\title{
Myrmica xavieri sp. n., a new ant species (Hymenoptera: Formicidae) from Spain
}

\author{
Alexander Radchenko, Graham W. Elmes \& *Riitta Savolainen
}

Radchenko, A., Elmes, G. W. \& Savolainen, R. 2008: Myrmica xavieri sp. n., a new ant species (Hymenoptera: Formicidae) from Spain. — Entomol. Fennica 19: 49-54.

Here we describe a new ant species from Spain, Myrmica xavieri. We place it in the Myrmica lobicornis species group of which three species are previously known in the Iberian Peninsula: M. lobicornis Nylander, M. wesmaeli Bondroit, and M. lobulicornis Nylander. Of these, M. xavieri most closely resembles $M$. wesmaeli. M. xavieri is likely an Iberian endemic, a relict of the fauna isolated in the Iberian Peninsula during the Pleistocene.

A. Radchenko, Museum and Institute of Zoology, Polish Academy of Sciences, 64 Wilcza Street,00-679 Warsaw, Poland; e-mail: agradchenko@hotmail.com

G. W. Elmes, Centre for Ecology \& Hydrology, CEH Wallingford, Crowmarsh Gifford, Wallingford, Oxfordshire, OX10 8BB, United Kingdom; e-mail: gwe@ceh.ac.uk

R. Savolainen, Department of Biological and Environmental Sciences, P.O. Box 65, 00014 University of Helsinki, Finland; *corresponding author's e-mail: riitta.savolainen@helsinki.fi

Received 19 January 2007, accepted 12 June 2007

\section{Introduction}

In the ant genus Myrmica, the shape of the antennal scape is important in defining species groups (Radchenko 1994a). Key features of the scape are the shape and orientation of any ridge or dentiform lobe at the bend of the base of the scape, often combined with a degree of bending. Some species have a vertical lobe at the bend of the scape (relative to the dorsal plane of the head), or a horizontal lobe or ridge, whereas in others the lobe or ridge is lacking or barely visible. Species of the Myrmica lobicornis group are unambiguously defined by the combination of following features of both the female and male castes (Radchenko 1994a, b): workers and queens have antennal scapes that are strongly curved and often angled at the base, and males have a relatively long scape, with scape index $\mathrm{SI}_{1}>0.60$ (see definition of the scape index below). In contrast, males of the schencki-group and scabrinodisgroup species - queens and workers of which are most easily confused with those of the lobicornis group - have a much shorter scape, with $\mathrm{SI}_{1}<$ 0.50 . The female castes of most lobicornis-group species have a vertical lobe or dent on the scape bend that never extends horizontally (relative to the dorsal plane of the head) as does the lobe or ridge of most scabrinodis-group species. At one extreme, lobicornis-group species with large lobes may be confused with some schencki-group species, and at the other extreme, species with no lobes may be confused with a few species of the scabrinodis group.

Three lobicornis-group species are previously known from the Iberian Peninsula: M. lobi- 
cornis Nylander, $M$. wesmaeli Bondroit, and $M$. lobulicornis Nylander which was raised to species rank by Seifert (2005). In the year 2000, one of us (RS) collected a nest of a lobicornis-group species, including sexual forms, which appeared distinct from the other three species. After a close examination of the Elmes Myrmica collection, another sample, collected ten years earlier, was detected. Individuals of the two nest samples, coming from well separated sites in Spain, showed the same clear morphological differences from all other known species, and therefore we describe them as a new species, Myrmica xavieri.

\section{Material and methods}

The material consisted of 20 workers, 30 queens (gynes), and 35 males from two nest series. The samples were collected by hand in Spain in 1990 and 2000. We measured 19 morphometric characters of workers, queens and males (unless otherwise stated), with an accuracy to $0.01 \mathrm{~mm}$, as follows:

$\mathrm{AH}$ height of alitrunk, measured from the upper level of mesonotum perpendicularly to the level of lower margin of mesopleuron (queens and males).

AL diagonal length of alitrunk seen in profile, from the neck shield to the posterior margin of the metapleural lobe (workers) and from the anterio-dorsal point of alitrunk to the posterior margin of the metapleural lobe (queens and males).

ESD distance between tips of propodeal spines from above (queens and workers).

ESL maximum length of propodeal spine in profile, measured along the spine from its tip to the deepest point of the propodeal constriction at the base of spine (queens and workers).

FLW maximum width between external borders of the frontal lobes (queens and workers).

FW minimum width of frons between the frontal carinae (queens and workers).

HL length of head in dorsal view, measured in a straight line from the anteriormost point of median clypeal margin (including any carinae or rugae, if they protrude over the anterior margin) to the mid-point of the occipital margin.

HTL length of hind tibia, measured from its junction with the femur to the junction with the first tarsal joint.

HW maximum width of head in dorsal view behind (above) the eyes.

PH maximum height of petiole in profile, measured from the uppermost point of the petiolar node perpendicularly to the imaginary line between the anteroventral (just behind the subpetiolar process) and posteroventral points of petiole.

PL maximum length of petiole from above, measured from the posterodorsal margin of petiole to its anterior edge at the articulation with propodeum; this can be difficult to see and petiole should be positioned such that measured points lay on the same plane, and as this is not always possible this variable is probably subject to greater measurement error than the others.

PNW maximum width of pronotum from above in dorsal view (workers).

PPH maximum height of postpetiole in profile from its uppermost to lowermost points, measured perpendicularly to the linear component of the lateral post-petiolar suture.

PPL maximum length of postpetiole, measured from above from its anterior to posterior margins.

PPW maximum width of postpetiole from above.

PW maximum width of petiole from above.

SCL combined length of scutum and scutellum from above (queens and males).

SCW maximum width of scutum from above (queens and males).

SL maximum straight-line length of antennal scape seen in profile.

We calculated the following indices based on the above morphometric characters:

$\mathrm{AI}=\mathrm{AL} / \mathrm{AH} \quad$ alitrunk length index

$\mathrm{CI}=\mathrm{HL} / \mathrm{HW} \quad$ cephalic index

$\mathrm{ESDI}=\mathrm{ESD} / \mathrm{ESL}$ epinotal (propodeal) spine

distance index

$\mathrm{ESLI}=\mathrm{ESL} / \mathrm{HW}$ epinotal (propodeal) spine length index 
Table 1. Means \pm standard deviations of the morphometric characters (see text for details) in $\mathrm{mm}$ for the holotype and paratypes of M. xavieri. Numbers of individuals measured per caste are given in parentheses in column headings.

\begin{tabular}{lllll}
\hline Character & Holotype & Workers $(20)$ & Queens $(7)$ & Males (9) \\
\hline AH & & & $1.106 \pm 0.034$ & $1.062 \pm 0.038$ \\
AL & 1.820 & $1.741 \pm 0.042$ & $1.923 \pm 0.035$ & $1.791 \pm 0.050$ \\
ESD & 0.460 & $0.445 \pm 0.021$ & $0.467 \pm 0.017$ & \\
ESL & 0.420 & $0.422 \pm 0.013$ & $0.491 \pm 0.019$ & \\
FLW & 0.430 & $0.426 \pm 0.013$ & $0.442 \pm 0.007$ & \\
FW & 0.340 & $0.333 \pm 0.011$ & $0.359 \pm 0.008$ & \\
HL & 1.220 & $1.209 \pm 0.027$ & $1.277 \pm 0.018$ & $0.896 \pm 0.019$ \\
HTL & 0.920 & $0.888 \pm 0.023$ & $0.943 \pm 0.018$ & $0.936 \pm 0.044$ \\
HW & 1.080 & $1.053 \pm 0.030$ & $1.121 \pm 0.026$ & $0.847 \pm 0.021$ \\
PH & 0.425 & $0.403 \pm 0.014$ & $0.461 \pm 0.007$ & $0.378 \pm 0.014$ \\
PL & 0.520 & $0.489 \pm 0.023$ & $0.560 \pm 0.013$ & $0.490 \pm 0.009$ \\
PNW & 0.800 & $0.757 \pm 0.023$ & & \\
PPH & 0.505 & $0.494 \pm 0.012$ & $0.566 \pm 0.011$ & $0.455 \pm 0.016$ \\
PPL & 0.420 & $0.382 \pm 0.022$ & $0.461 \pm 0.012$ & $0.356 \pm 0.016$ \\
PPW & 0.475 & $0.471 \pm 0.015$ & $0.556 \pm 0.009$ & $0.446 \pm 0.016$ \\
PW & 0.330 & $0.324 \pm 0.013$ & $0.360 \pm 0.014$ & $0.301 \pm 0.015$ \\
SCL & & & $1.276 \pm 0.024$ & $1.204 \pm 0.026$ \\
SCW & 1.000 & $0.976 \pm 0.019$ & $0.920 \pm 0.013$ & $0.867 \pm 0.017$ \\
SL & & $0.993 \pm 0.010$ & $0.839 \pm 0.018$ \\
\hline
\end{tabular}

$\mathrm{FI}=\mathrm{FW} / \mathrm{HW} \quad$ frons index

$\mathrm{FLI}=\mathrm{FLW} / \mathrm{FW} \quad$ frontal lobe index

$\mathrm{HTI}=\mathrm{HTL} / \mathrm{HW}$ hind tibia length index

$\mathrm{PI}_{1}=\mathrm{PL} / \mathrm{PH} \quad$ petiole index (1)

$\mathrm{PI}_{2}=\mathrm{PL} / \mathrm{HW} \quad$ petiole index (2)

$\mathrm{PPI}_{1}=\mathrm{PPL} / \mathrm{PPH}$ postpetiole index (1)

$\mathrm{PPI}_{2}=\mathrm{PPH} / \mathrm{PPW}$ postpetiole index (2)

$\mathrm{PPI}_{3}=\mathrm{PPW} / \mathrm{PW}$ postpetiole index (3)

$\mathrm{PPI}_{4}=\mathrm{PPW} / \mathrm{HW}$ postpetiole index (4)

$\mathrm{SCI}=\mathrm{SCL} / \mathrm{SCW}$ scutellum index

$\mathrm{SI}_{1}=\mathrm{SL} / \mathrm{HL} \quad$ scape index (1)

$\mathrm{SI}_{2}=\mathrm{SL} / \mathrm{HW} \quad$ scape index (2)

\section{Description of Myrmica xavieri sp. $\mathrm{n}$. (Figs. 1-3, Tables 1-2)}

Material examined. Holotype worker: Spain, Aragón, Province of Teruel, Sierra de Albarracín, Fuente del Canto, ca. three km WSW of Bronchales $\left(40^{\circ} 30^{\prime} \mathrm{N}, 1^{\circ} 37^{\prime} \mathrm{W}, 1,630 \mathrm{~m}\right.$ a.s.1.), 1.VIII. 2000, No. 334/2000 (series E-186 in the collection of G. W. Elmes), leg. R. Savolainen and K. Vepsäläinen. Paratypes: 9 workers, 30 gynes and 35 males from the nest of the holotype; 10 workers, Spain, Castilla y Léon, Province of Soria, Sierra de Urbion, ca. $25 \mathrm{~km} \mathrm{~N}$ of Soria (from memory Elmes places it in the region $42^{\circ} \mathrm{N}, 2^{\circ} 30^{\circ} \mathrm{W}$,
1,500 m a.s.1.), 7.VII.1990, series E-117, leg. G. W. Elmes. The nest of the holotype was in open pine forest (Pinus sylvestris) with moist soil. The paratype nest was ca. $180 \mathrm{~km} \mathrm{NNW}$ of the holotype site in well-trampled grass on a mountain pass near a pine forest in the mountains of Soria Province, by a road between Soria and Logroño. Both nests were in soil under stones. The holotype is deposited in the Natural History Museum (BMNH), London, United Kingdom, and the paratypes, in the Institute of Zoology of Ukrainian National Academy of Sciences, Kiev, Ukraine, and in the collections of Graham W. Elmes, United Kingdom, and Xavier Espadaler, San Cugat, Spain.

Diagnosis. Worker (Fig. 1). Head longer than its width with slightly convex sides and occipital margin, and broadly rounded occipital corners. Anterior clypeal margin narrowly rounded, somewhat prominent, with no medial notch. Frontal carinae fairly strongly curved, frons relatively narrow, though frontal lobes not widely extended. Antennal scape strongly angled at the base, with small vertical lobe.

Alitrunk with slightly convex promesonotal dorsum; promesonotal suture clearly visible from above; metanotal groove sharp and deep. Propodeal spines long, acute, straight and diver- 


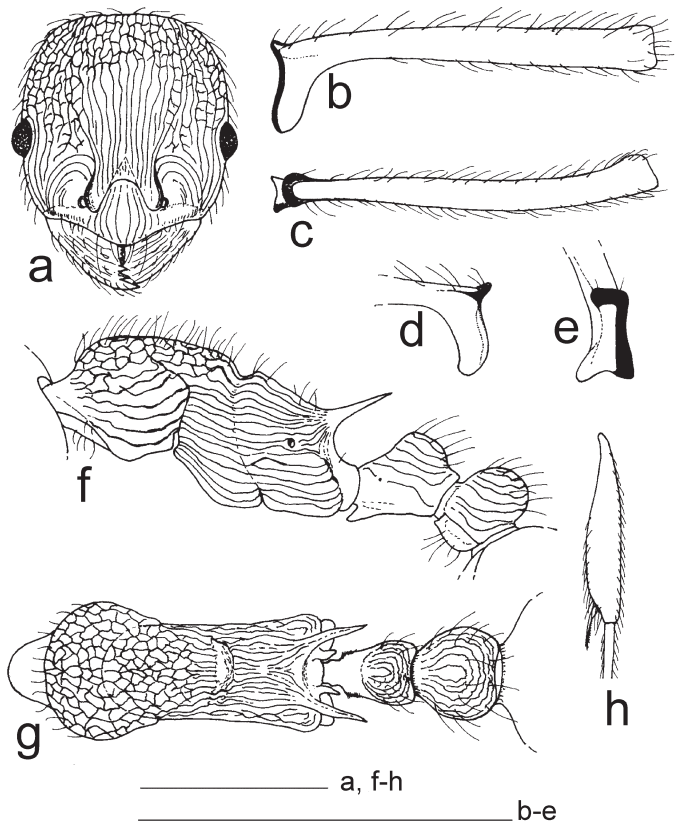

Fig. 1. Myrmica xavieri worker (holotype; scale bars 1 $\mathrm{mm}$ ). - a. Head, dorsal view. - b. Scape, lateral view, seen from behind. - c. Scape, dorsal view. - d. Base of scape, lateral view, seen from ahead. - e. Base of scape, anterior view. - f. Alitrunk and waist in profile. - g. Alitrunk and waist from above. - h. Tibia and base of first tarsal joint of hind leg.

gent (seen from above) projecting backwards at an angle less than $45^{\circ}$ (seen in profile). Petiole with distinct anterior peduncle, its anterior face slightly concave, not steep, meeting the dorsal surface at a blunt rounded angle; dorsal surface of petiolar node slightly convex, gradually sloping posteriorly. Postpetiole distinctly shorter than broad, with convex dorsum. Spurs on middle and hind tibiae well developed and pectinate.

Frons with sinuous longitudinal rugae, remaining parts of head dorsum with coarse reticulation. Antennal sockets surrounded by concentric rugae. Clypeus with longitudinal rugae, frontal triangle coarsely striated. Sides of alitrunk with moderately coarse sinuous longitudinal rugae, pronotum with coarser rugae and reticulation. Petiole and postpetiole with fairly coarse longitudinally-concentric rugae, more sinuous on the nodes of dorsum. Surface between rugae on the body smooth and shiny.

Head margins with abundant, semierect hairs; alitrunk dorsum and waist with numerous hairs

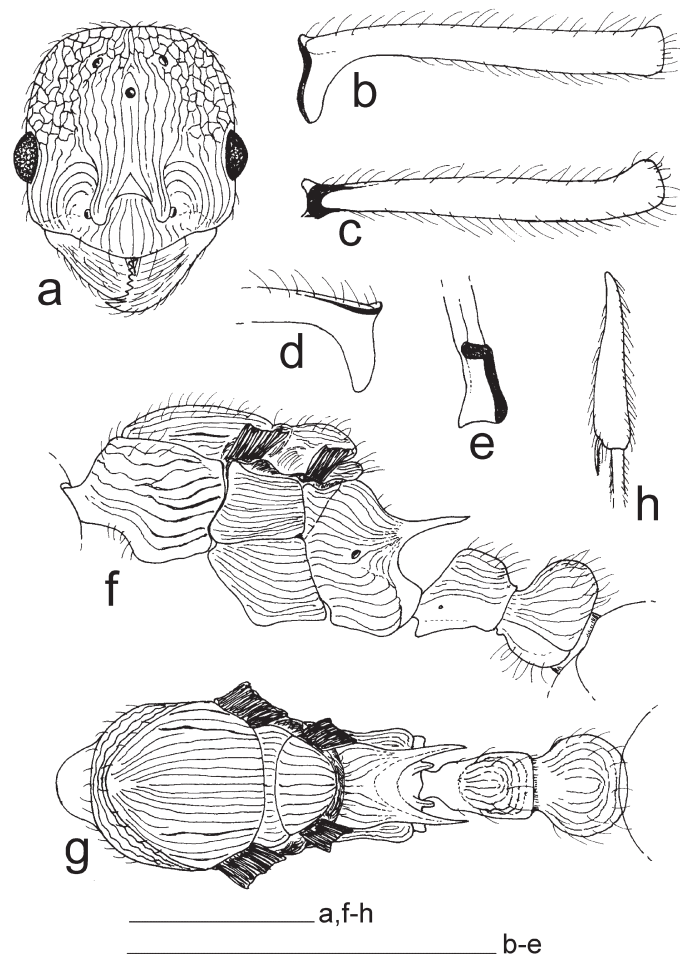

Fig. 2. Myrmica xavieri queen (paratype; scale bars 1 $\mathrm{mm}$ ). - a. Head, dorsal view. - b. Scape, lateral view, seen from behind. - c. Scape, dorsal view. - d. Base of scape, lateral view, seen from ahead. - e. Base of scape, anterior view. - f. Alitrunk and waist in profile. $-\mathrm{g}$. Alitrunk and waist from above. $-\mathrm{h}$. Tibia and base of first tarsal joint of hind leg.

much longer than those on head margin. General colour reddish-brown, appendages somewhat lighter.

Queen (Fig. 2). Like worker in shape of head, scape, general colour and pilosity of the body, but with coarser sculpture, especially on the alitrunk, petiole and postpetiole than the worker. The main difference is a more massive petiole than in workers, with a shorter anterior peduncle. Also the propodeal spines are longer and less divergent than in workers.

Male (Fig. 3). Head somewhat longer than broad, with slightly convex sides and occipital margin, and gradually rounded occipital corners. Anterior clypeal margin narrowly rounded and slightly prominent with no medial notch. Antennal scape very long and strongly curved at the base. Antennae 13-segmented, with 3-segmented club, second funicular segment about twice as 


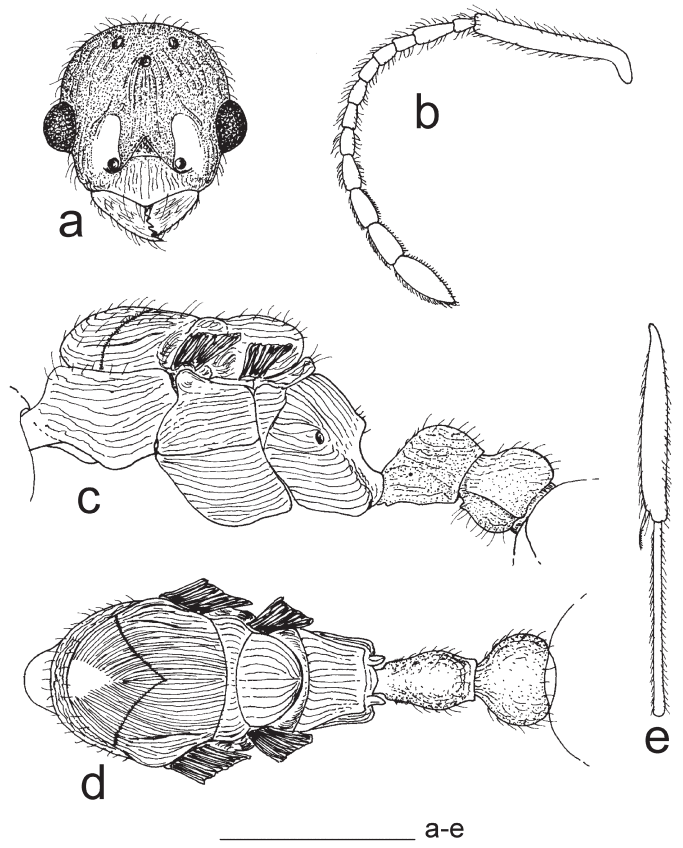

Fig. 3. Myrmica xavieri male (paratype; scale bar 1 $\mathrm{mm}$ ). - a. Head, dorsal view. - b. Antenna. - c. Alitrunk and waist in profile. - d. Alitrunk and waist from above. - e. Tibia and first tarsal joint of hind leg.

long as the third one.

Alitrunk fairly long, scutum slightly convex, scutellum not projecting dorsally above scutum when seen in profile. Propodeum with blunt rounded tubercles. Petiole rather massive, relatively high, with straight anterior face and rounded dorsum of node; postpetiole distinctly longer than its width, with weakly convex dorsum.

Head dorsum with striation and dense punctures. Scutum with dense longitudinally-concentric rugae, scutellum with coarser and less abundant rugae. Pro- and mesopleura with fine rugae, propodeum with coarser rugae. Petiole and postpetiole finely but not densely punctured, petiole additionally has fine rugulae, central part of petiolar and postpetiolar dorsum smooth and shiny. Surface on alitrunk between rugae smooth and shiny.

Head margins, alitrunk and waist with abundant, relatively short semierect hairs. Scape, tibiae and tarsi with short subdecumbent to suberect hairs. General colour of body dark brown to black, appendages somewhat lighter.
Etymology. This species is dedicated to the Spanish myrmecologist, Dr. Xavier Espadaler.

\section{Discussion}

Differential diagnosis. The female castes of $M$. xavieri clearly differ from those of the other lobicornis-group species by the absence of a medial notch in their anterior clypeal margin which is narrowly rounded and prominent. All the other species have a distinct medial notch in the anterior clypeal margin which is broadly rounded and not prominent. Superficially $M$. xavieri may be confused with $M$. lobulicornis and especially with $M$. wesmaeli. The workers of $M$. xavieri differ from both, however, by their distinctly narrower frons (mean FI 0.32 vs. 0.34 and 0.37 , respectively). When the 20 specimens measured here (Table 2) were compared with a randon sample of $M$. wesmaeli (which included the lectotype and several paralectotypes) in a linear discriminant analysis, FI alone was sufficient to produce $100 \%$ discrimination between the two samples (linear discriminant functions were 2905 FI - 534 for M. wesmaeli and 2487 FI - 391 for M. xavieri ). Inclusion of $\mathrm{SI}_{2}$ and $\mathrm{HTI}$ somewhat improved the discrimination (the three indices are morphometrics standardised for size by dividing by HW).

M. xavieri has distinctly more extended frontal lobes than M. wesmaeli (mean FLI 1.28 vs. 1.18 ), but less extended frontal lobes than $M$. lobulicornis (mean FLI 1.34). M. xavieri also differs from M. lobicornis and M. lobulicornis by its distinctly longer propodeal spines (mean ESLI $0.40 v s . \leq 0.30)$ and by the different shape of the petiole: the anterior peduncle of the petiole is very short in M. lobicornis and M. lobulicornis, and the anterior face of the node (seen in profile) is flat and steep, meeting the dorsal surface at a right-angle or even more acutely. In M. xavieri the peduncle is longer, its anterior face more concave, meeting the dorsal surface with a blunt rounded angle.

Like the workers and queens, the males of $M$. xavieri clearly differ from all other species of the lobicornis group by their narrowly rounded and unnotched anterior clypeal margin. They also differ by having a distinctly longer antennal scape 
Table 2. Means \pm standard deviations of the indices calculated from the morphometric characters of Table 1 (see text for details) for the holotype and paratypes of $M$. xavieri. Numbers of individuals measured per caste are given in parentheses in column headings.

\begin{tabular}{lllll}
\hline Character & Holotype & Workers $(20)$ & Queens $(7)$ & Males (9) \\
\hline $\mathrm{Al}$ & & & $1.740 \pm 0.039$ & $1.688 \pm 0.062$ \\
$\mathrm{Cl}$ & 1.130 & $1.149 \pm 0.014$ & $1.139 \pm 0.017$ & $1.058 \pm 0.018$ \\
$\mathrm{ESDI}$ & 1.095 & $1.056 \pm 0.056$ & $0.952 \pm 0.044$ & \\
$\mathrm{ESLI}$ & 0.389 & $0.401 \pm 0.016$ & $0.438 \pm 0.014$ & \\
$\mathrm{FI}$ & 0.315 & $0.316 \pm 0.009$ & $0.320 \pm 0.004$ & \\
$\mathrm{FLI}$ & 1.265 & $1.281 \pm 0.023$ & $1.231 \pm 0.023$ & \\
$\mathrm{HTI}$ & 0.852 & $0.843 \pm 0.019$ & $0.841 \pm 0.023$ & $1.105 \pm 0.051$ \\
$\mathrm{PI}_{1}$ & 1.224 & $1.214 \pm 0.028$ & $1.215 \pm 0.020$ & $1.296 \pm 0.033$ \\
$\mathrm{Pl}^{1}$ & 0.481 & $0.464 \pm 0.018$ & $0.500 \pm 0.012$ & $0.579 \pm 0.015$ \\
$\mathrm{PPI}_{1}$ & 0.832 & $0.772 \pm 0.035$ & $0.815 \pm 0.021$ & $0.781 \pm 0.013$ \\
$\mathrm{PPI}^{1}$ & 1.063 & $1.048 \pm 0.032$ & $1.020 \pm 0.025$ & $1.022 \pm 0.025$ \\
$\mathrm{PPI}^{2}$ & 1.439 & $1.455 \pm 0.041$ & $1.546 \pm 0.066$ & $1.481 \pm 0.046$ \\
$\mathrm{PPI}^{3}$ & 0.440 & $0.448 \pm 0.010$ & $0.496 \pm 0.013$ & $0.527 \pm 0.024$ \\
$\mathrm{SCl}^{4}$ & & & $1.387 \pm 0.030$ & $1.390 \pm 0.022$ \\
$\mathrm{SI}^{1}$ & 0.820 & $0.807 \pm 0.013$ & $0.778 \pm 0.013$ & $0.937 \pm 0.020$ \\
$\mathrm{SI}_{2}$ & 0.926 & $0.927 \pm 0.018$ & $0.886 \pm 0.023$ & $0.991 \pm 0.022$ \\
\hline
\end{tabular}

$\left(\mathrm{SI}_{1}>0.90, \mathrm{SI}_{2}>0.95 v s .<0.85,<0.90\right)$ and thus the male may be confused more easily with $M$. rubra (L.) or M. ruginodis Nylander. The scape of the latter two species is, however, gently curved at the base, whereas that of $M$. xavieri is strongly angled.

Notes. We also identified two additional workers and two males (probably from a single nest) ca. $65 \mathrm{~km} \mathrm{SE}$ of the holotype site, collected by Cedric A. Collingwood in Spain (Aragón, Province of Teruel, Sierra de Javalambre, Javalambre, 1,500-1,800 $\mathrm{m}$ a.s.1. (inferred from maps), June 1986, series E-178 in the collection of G. W. Elmes) as M. xavieri. The males are indistinguishable from those described above. The workers are similar, but exhibit minor differences in the shape of petiole and by one worker having a smoother, the other a coarser sculpture than the workers described above, and by distinctly more extended frontal lobes (FLI 1.35-1.36). In the discriminant analysis (above) the two workers fell within the $M$. xavieri cluster, indicating larger morphological variation of $M$. xavieri than observed in the two type series. To prevent possible confusion in the future, we did not include these specimens in the type series.

We place M. xavieri in the lobicornis species group, because many of the features of both the female and male castes best match those of that group compared to any other species group. The absence of a notched clypeal margin in both castes and the exceptionally long scapes of the males, however, well separate $M$. xavieri from all other known species of that group (ca. 20 known species). It is most probably an Iberian endemic, a relict of the fauna isolated in the Iberian Peninsula during the Pleistocene.

Acknowledgements. We are grateful to Cedric A. Collingwood for providing us with material, and Kari Vepsäläinen, Bernhard Seifert, and Judith Wardlaw for making useful comments on the manuscript. This work was supported by the grant of the Ministry of High Education and Sciences of Poland (No. 2P04C 064 29) to AR, and the Academy of Finland to RS.

\section{References}

Radchenko, A. G. 1994a: Taxonomic structure of the genus Myrmica (Hymenoptera, Formicidae) of Eurasia. Communication I. — Zoologicheskiy Zhurnal 73: 3951 [in Russian; English translation Entomol. Rev. 1995, 74: 91-106].

Radchenko, A. G. 1994b: Survey of the lobicornis group of the genus Myrmica (Hymenoptera, Formicidae) of the Central and Eastern Palaearctic. - Zoologicheskiy Zhurnal 73: 81-92 [in Russian; English translation, Entomol. Rev. 1995, 74: 133-146].

Seifert, B. 2005: Rank elevation in two European ant species: Myrmica lobulicornis Nylander, 1857, stat. n. and Myrmica spinosior Santschi, 1931, stat. n. (Hymenoptera: Formicidae). - Myrmecologische Nachrichten 7: 1-7. 